OPEN

SUBJECT AREAS:

GLASSES

PHASE TRANSITIONS AND CRITICAL PHENOMENA

Received

11 November 2014

Accepted

13 January 2015

Published

9 February 2015

Correspondence and requests for materials should be addressed to

J.C.M.-G.

(jmartinezgarcia@ gmail.com) or S.J.R.

(sylwester.rzoska@ gmail.com)

* Current address: Adolphe Merkle Institute Chemin des Verdiers $4 \mathrm{CH}-1700$, Fribourg Switzerland.

\section{Fragility and basic process energies in vitrifying systems}

\author{
Julio Cesar Martinez-Garcia ${ }^{1 *}$, Sylwester J. Rzoska ${ }^{2,3}$, Aleksandra Drozd-Rzoska², Szymon Starzonek ${ }^{3}$ \\ \& John C. Mauro ${ }^{4}$
}

${ }^{1}$ Department of Chemistry and Biochemistry, University of Berne, Freiestrasse 3, Berne CH-3012, Switzerland, ${ }^{2}$ Institute of High Pressure Physics Polish Academy of Sciences, ul. Sokołowska 27/39, 01-142 Warsaw, Poland, ${ }^{3}$ Institute of Physics \& ŚMCEBI, University of Silesia, ul. 75 Pułku Piechoty 1A, 41-500 Chorzów, Poland, ${ }^{4}$ Science and Technology Division, Corning Incorporated, Corning, New York 14831, USA.

The concept of 'fragility' constitutes a central point of the glass transition science serving as the 'universal' metric linking previtreous dynamics of qualitatively distinct systems. Finding the fundamental meaning of fragility is the 'condicio sine qua' for reaching the long expected conceptual breakthrough in this domain. This report shows that fragility is determined by the ratio between two fundamental process energies, viz. the activation enthalpy and activation energy. The reasoning, avoiding any underlying physical model, is supported by the experimental evidence ranging from low molecular weight liquids and polymers to plastic crystals and liquid crystals. All these lead to the new general scaling plot for dynamics of arbitrary glass former. The limited adequacy of broadly used so far semi-empirical relationships between fragility and the activation energy is shown. Results presented remain valid for an arbitrary complex system and collective phenomena if their dynamics is described by the general super-Arrhenius relation.

G lass transition constitutes one of grand challenges of condensed and soft matter physics as well as modern materials science ${ }^{1-4}$, where a long-expected fundamental breakthrough could lead to innovative implementations ranging from silicate glasses ${ }^{5}$ and plastics ${ }^{6}$ to pharmaceuticals ${ }^{7}$ and foods $s^{8}$. The ultimate progress in this area is also important for biotechnological ${ }^{9}$, geophysical ${ }^{10}$, metallurgical ${ }^{11}$ and electronic devices ${ }^{12}$ implementations. Notable is the fact that glass transition physics is considered as one of key references for collective phenomena science, aimed to discover properties emerging from complex correlations ${ }^{13}$.

Of particular interest of the glass transition research is the identification of universal features in the previtreous dynamic regime shared amongst a surprising variety of systems including low molecular weight liquids, polymers, liquids crystals, plastic crystals, colloids, metallic alloys, silicates, spin glasses, etc ${ }^{2-5}$. A key metric linking so distinct glass formers was introduced by Austen Angell ${ }^{14}$, basing on a master plot of $\log _{10} \eta(T)$ and $/$ or $\log _{10} \tau(T)$ versus $T_{g} / T$ where $\eta(T)$ stands for viscosity, $\tau(T)$ for structural (primary) relaxation time and $T_{g}$ is the glass temperature ${ }^{14,15}$. This was possible due to the empirical normalization assumption for the glass transition temperature $\eta\left(T_{g}\right)=10^{13}$ Poise and $\tau\left(T_{g}\right)=100 \mathrm{~s}$. Subsequently, a metric describing the slope for $T \rightarrow T_{g}$, called 'fragility', was proposed ${ }^{14,15}$ :

$$
m=m\left(T_{g}\right)=\left[\frac{d \log _{10} \eta(T)}{d\left(T_{g} / T\right)}\right]_{T=T_{g}} \quad \text { or } \quad m=m\left(T_{g}\right)=\left[\frac{d \log _{10} \tau(T)}{d\left(T_{g} / T\right)}\right]_{T=T_{g}}
$$

The fragility index $m$ describes the degree of shifting from the basic Arrhenius dynamics to the super-Arrhenius (SA) one, described by the general form²:

$$
\tau(T)=\tau_{0} \exp \left(\frac{\Delta E_{a}(T)}{R T}\right) \quad \text { or } \quad \eta(T)=\eta_{0} \exp \left(\frac{\Delta E_{a}(T)}{R T}\right)
$$

where $T>T_{g}, R$ denotes the gas constant and $\Delta E_{a}(T)$ the apparent activation energy. The basic Arrhenius dependence is restored for $\Delta E_{a}(T)=E_{a}=$ const.

There are two general types of glass formation defined by the fragility metric: (i) 'fragile' systems with highly SA dynamics $(m>50)$ and (ii) 'strong' ones, with close-to-Arrhenius behavior $(m<30)^{2,14,15}$. The basic Arrhenius behavior $\tau(T)=\tau_{0} \exp \left(E_{a} / R T\right)$ is associated with the minimal value of the fragility index and most often related to $m$ $=\log _{10}\left(\tau\left(T=T_{g}\right)\right)-\log _{10} \tau_{0}=2+14=16$, i.e. assuming for the prefactor $\tau_{0}=10^{-14} s$ in the SA eq. $(2)^{2,15}$. Notwithstanding, for silicate liquids extremely strong SA behavior with a minimal $m=14.93$ was found ${ }^{16}$. 
Experimental estimations of the prefactor in the SA equation ranges from $\sim 10^{-11} s$ to even $\sim 10^{-18} s^{2,17}$, what indicates on the systemdependent minimal fragility.

Qualitative mapping of the previtrous increase of relaxation times or viscosity onto a single chart has led to the concept of fragility, becoming a focal point for research in glass transition physics ${ }^{2,3}$. The most important appeared as the link between two basic properties, viz. fragility and the activation energy ${ }^{2,3,18-20}$. One may claim that the ultimate explanation of this problem is the "condicio sine qua" for reaching the conceptual breakthrough in glass transition physics ${ }^{2,3}$. Surprisingly, despite decades of studies the situation is puzzling.

The first and broadly implemented up to now dependence ${ }^{20-26}$ was proposed by Boehmer et al. ${ }^{15}$ in 1993:

$$
\Delta E_{a}\left(T_{g}\right)=\Delta E_{a}\left(T / T_{g} \rightarrow 1\right)=R T_{g} m \ln 10
$$

In 2004, Novikov and Sokolov ${ }^{27}$ proposed yet another relation, supported by experimental evidence for a set of glass forming liquids ${ }^{27,28}$ :

$$
\Delta E_{a}\left(T_{g} / T \rightarrow 0\right)=\frac{(19.2)^{2} T_{g} \ln 10}{m}
$$

This report presents the critical discussion of eqs. (3) and (4) and shows that their validity is casual. Subsequently, it presents the lacking so far fundamental link between fragility and fundamental process energies, viz. the activation energy and the activation enthalpy. The new, 'ultimate', scaling relation linking fragility and the activation energy has been also derived. Analytic results are supported by the clear experimental evidence for a broad range of glass formers.

\section{Results}

The new insight into fragility of glass formers. In Refs. 29, 30 the new approach for the insight into dynamics of the previtreous domain, based solely on the SA eq. (2) and the metric describing relative changes of the apparent activation energy was introduced:

$$
I_{D O}(T)=-\frac{d \Delta E_{a}(T) / \Delta E_{a}(T)}{d T / T}=-\frac{d \ln \Delta E_{a}(T)}{d \ln T}
$$

The analysis in Refs. 29, 30 was possible due to the innovative way of determining $\Delta E_{a}(T)$, which avoids the biasing impact of generally unknown prefactor $\tau_{0}$ in the SA eq. (2). This model-free approach lead to a set of notable findings including the limited fundamental adequacy of the Vogel-Fulcher-Tamman (VFT) ${ }^{31-33}$ equation, identifying the role of local symmetry in glass formation and showing the ultimate way of "dynamic" estimation of the ideal glass transition temperature ${ }^{29,30}$.

Linking the SA eq. (2) and eq. (5) for the apparent activation energy temperature index one obtains:

$$
\begin{aligned}
\Delta H_{a}^{\prime}(T) & =\frac{\Delta H_{a}(T)}{R}=\frac{\partial \ln \tau(T)}{\partial(1 / T)} \\
& =\left[\Delta E_{a}^{\prime}(T)+\frac{1}{T} \frac{\partial \Delta E_{a}^{\prime}(T)}{\partial(1 / T)}\right]=\Delta E_{a}^{\prime}(T)\left[1-I_{D O}(T)\right]
\end{aligned}
$$

where the identity $d(1 / T)=(-(1 / T) d T)(1 / T)=-(d \ln T)(1 / T)$ was used, $\Delta H_{a}(T)$ denotes the apparent activations enthalpy (see also Ref. 17 and Suppl. Info of Refs. 29, 30 where clear derivations of the relationship between $\Delta E_{a}(T), \Delta H_{a}(T)$ and $d \ln \tau(T) / d(1 / T)$ are given), $\Delta E_{a}^{\prime}(T)=\Delta E_{a} / R$.

The above dependence directly yields:

$$
I_{D O}(T)=\frac{\Delta H_{a}(T)}{\Delta E_{a}(T)}-1
$$

Figure 1 shows that eq. (7) can serve as the base for the new "universal scaling plot" for the previtreous dynamics of arbitrary glass former. It includes representatives from LMW, P, ODIC and LC categories ${ }^{29,30,34-39}$. Notable is the correlation with the classical 'Angell' plot ${ }^{2,3,14,15}$, presented in the inset for the same systems. For both plots the increase of curvature indicates the rise of fragility. However, in the main plot fragility it is directly determined by the ratio of fundamental process energies, namely:

$$
I_{D O}(T)=\left(\frac{T_{g}}{T}\right) \frac{d \ln \Delta E_{a}(T)}{d(T g / T)}
$$

which lead to:

$$
M=I_{D O}\left(T_{g}\right)=\left.\frac{d \ln \Delta E_{a}(T)}{d(T g / T)}\right|_{T=T g}=\frac{\Delta H_{a}\left(T_{g}\right)}{\Delta E_{a}\left(T_{g}\right)}-1
$$

The onset of the Arrhenius behavior is associated with the negligible curvature and almost horizontal behavior for $\Delta H_{a}(T) / \Delta E_{a}(T) \ll 1$ in Fig. 1.

The classical fragility index $m$ (eq. (1)) is still explained as the "formal" parameter, namely the slope at the 'Angell' plot ${ }^{2,14,15}$. The new fragility parameter $M=I_{D O}\left(T_{g}\right)$ gives directly value between two basic process energies, which are then key fundamental features determining the value of fragility. Using eqs. (1), (2) and (9) one obtains the link between the 'classical' $(m)$ and new $(M)$ fragility metrics:

$$
m=C(1+M)=C\left(1+I\left(T_{g}\right)\right)=C \frac{\Delta H_{a}\left(T_{g}\right)}{\Delta E_{a}\left(T_{g}\right)}
$$

where the constant $C=2-\log _{10}\left(\tau_{0}\right)=13-18$.

The relationship between $m$ and $I_{D O}\left(T_{g}\right)$ was indicated earlier by Hecksher et al. ${ }^{40}$, but without an explanation of the physical meaning of $I_{D O}\left(T_{g}\right)$.

The experimental confirmation of the behavior predicted by eq. (10) is given in Figure 2. It is notable that eq. (10), showing also the link of $m$ to basic process energies, indicates also the uncertainty introduced by the prefactor $\tau_{0}$ or $\eta_{0}$ in SA eq. (2). The summary of characteristics for aforementioned experimental systems is given in Table 1.

However, the most fundamental eqs. (1) and (2) directly indicate that the increasing SA behavior is associated with the rising nonlinearity at the "Arrhenius-type" plot $\ln \left(\Delta E_{a}(T)\right)$ vs. $1 / T$. Fig. 3 presents such plot, revealing the lack of a correlation between the increasing curvature of the apparent activation energy, coupled to rising fragility $m$, and the value of $\Delta E\left(T_{g}\right)$. This is in clear disagreement with mentioned above basic prediction (eq. (3)). Moreover, the simply linearization based on eqs. (1) and (2) yields $\ln \left\lfloor\Delta E_{a}\left(T_{g}\right) / T_{g}\right\rfloor$ $=\ln [m]+c t e$, i.e. the linear function with the intercept at cte $=\ln [R$ $\ln 10]>0$ and the directional factor $b=1$. Such prediction is anticorrelated with experimental data, as shown in the inset in Fig. 3 via the dashed line. Consequently, the used so far basic link between the activation energy and fragility $\Delta E_{a}\left(T_{g}\right)=R T_{g} m \ln 10$, i.e. (eq. $(3))^{2,14,16,20-26}$, is inherently invalid.

However, the simple analysis based solely on general eqs. (1) and (2) and eq. (9), derived above yields (see also Methods section):

$$
\begin{aligned}
\Delta E_{a}^{N}\left(T_{g}\right) & =R T_{g} m \ln 10-\left(\left.\frac{\partial \Delta E_{a}^{\prime}(T)}{\partial\left(T_{g} / T\right)}\right|_{T=T_{g}}\right) \\
& =\frac{R T_{g} m \ln 10}{I_{D O}\left(T_{g}\right)+1}=\frac{R T_{g} m \ln 10}{M+1}
\end{aligned}
$$

Figure 4 shows that the implementation of eq. (11) orders "chaotically scattered" curves in the main part of Fig. 3. It also leads to the superior agreement with experimental data given the inset in Fig. 3 (the solid line). Consequently, eq. (11) can be considered as the new ultimate link between the activation energy and fragility, valid for an arbitrary glass former. 


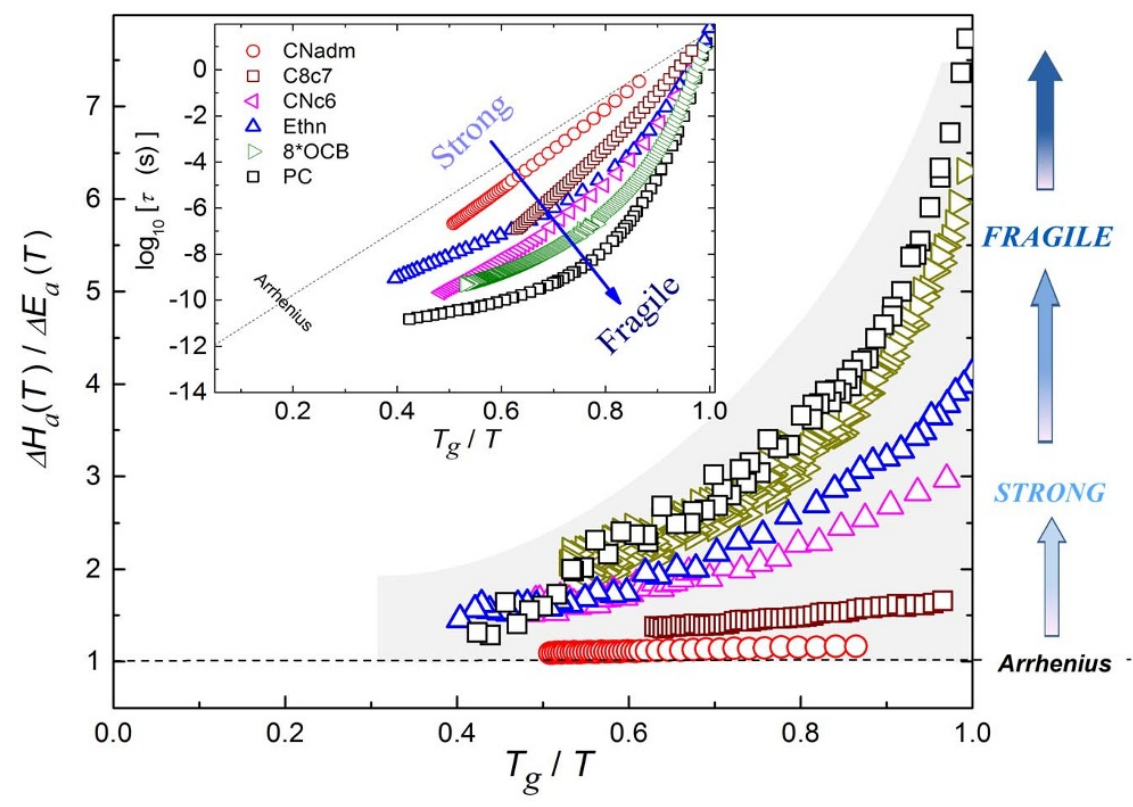

Figure 1 The evolution of the ratio between the apparent activation enthalpy and energy for selected glass forming systems. The inset shows the classic "Angell plot" ${ }^{\prime, 14,15}$ for $\tau(T)$ experimental data, constituting the base for determining non-biased ratio of process energies in the main plot. For basic data see Table 1.

Novikov and Sokolov ${ }^{27,28}$ proposed yet another relation linking fragility (determined for $T_{g} / T \rightarrow 1$ ) with the activation energy but this time taken for $T_{g} / T \rightarrow 0$, viz. eq. (4). For explaining its meaning, let's recall that in the low temperature domain $\left(T_{g} / T \rightarrow 1\right)$ the increase of curvature and the slope in the 'Angell' plot ${ }^{14,15}$ is linked to increasing fragility $m$. For the same plot, $\log _{10} \eta(T)$ or $\log _{10} \tau(T)$ vs. $T_{g} / T$, in the high temperature domain the decreasing slope is related to increasing value of $m$. This behavior is illustrated in Figure 5, showing that Novikov and Sokolov ${ }^{27,28}$ relation $\Delta E_{a}\left(T_{g} / T \rightarrow 0\right) \sim$ $1 / m$ (eq. (4)) results from the construction of the 'Angell' plot. The underlying assumption of eq. (4) is also the "universal" value of the viscosity $\left(\log _{10} \eta_{0}=-4\right)$ or for relaxation time $\log _{10} \tau_{0}=-14$ for $T_{g} / T \rightarrow 0$. This border values are considered as hypothetical universal values of the prefactor in the SA eqs. (1).

However, the existence of such universal values of prefactors can be questioned, particularly when taking into account different categories of glass formers, as discussed above. All these indicate on the

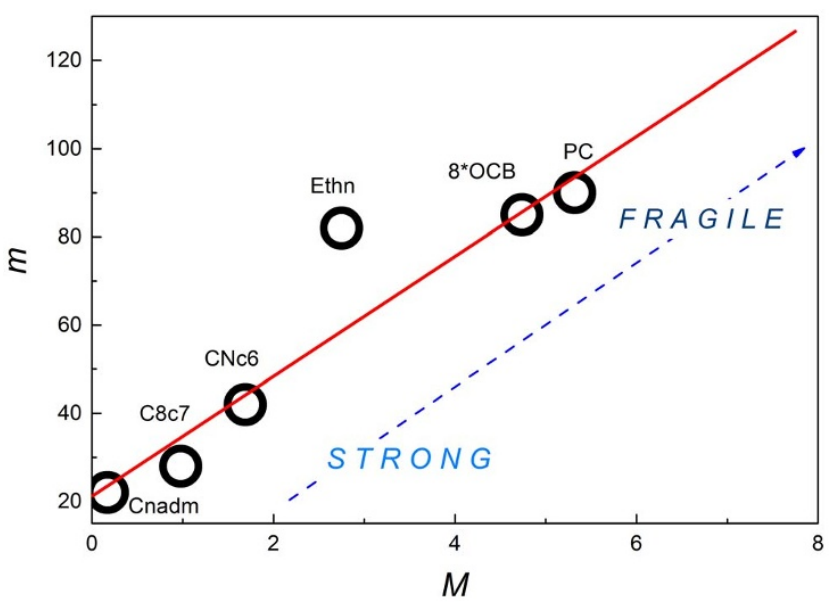

Figure $2 \mid$ The experimental tests of the relationship between the 'classical fragility metric $m$ and the new metric $M=\Delta H_{a}\left(T_{g}\right) / \Delta E_{a}\left(T_{g}\right)-$ 1. Results are for selected glass forming LMW, P, ODC and LC systems (see Table 1). necessity of a supplementary analysis, related to: (i) the activation energy determined without the biasing impact of the prefactor and (ii) the impact of the qualitative differences between dynamic the high- and low- temperatures domains. The latter is particularly important, because eq. (4) suggests direct causal link between different dynamic domains.

Following the above discussion, as well as the evidence from Refs. $2,29,30$, one can indicate following basic features of the ultraslowing/ultraviscous domain:

- There are no glass forming systems in the ultraviscous/ultrasowing domain where $\Delta E_{a}(T)$ decreases on cooling, i.e. $\forall_{T_{g}<T<T_{\infty}}$ $\partial \Delta E_{a}(T) / \partial(1 / T) \geq 0$ and $I_{D O}(T) \geq 0$.

- For extremely strong glass formers $\Delta E_{a}(T) \sim \Delta H_{a}(T)$ for the whole low temperature dynamic domain. In the case of the Arrhenius behavior $\Delta E_{a}(T)=\Delta H_{a}(T)=$ const.

- In the ultraviscous/ultraslowing domain always $\Delta H_{a}(T)>$ $\Delta E_{a}(T)$ and in the vicinity of $T_{g}$ even $\Delta H_{a}(T) \gg \Delta E_{a}(T)$. This is particularly evident for fragile ultraslowing and/or ultraviscous systems.

\section{Discussion}

The fragility and the activation energy are the most fundamental characteristics of glass transition. One can expect that a conceptual progress in this challenging area of condensed matter physics needs the unequivocal relationship between these quantities. However, this basic problem appeared to be surprisingly difficult, viz. the title of the recent Ref. 42: "The fragility and other properties of glass-forming liquids: Two decades of puzzling correlations".

The current report presents the first ever evidence of the unequivocal link between fragility and ratio of two basic process energies: the activation energy and the activation enthalpy in the low temperature ultraviscous/ultraslowing dynamic domain. It is worth recalling that the activation energy $\Delta E_{a}(T)$ is associated with the energy barrier necessary to boost a process, i.e. a transition state's free energy (the energy barrier) minus the energy of substrate's. This report shows the direct link of fragility to the ratio of these energies: $m=\left(2-\log _{10}\right.$ $\left.\tau_{0}\right)\left(\Delta H_{a}\left(T_{g}\right) / \Delta E_{a}\left(T_{g}\right)\right)$, but affected by the uncertainty associated with the SA prefactor $\tau_{0}$ (or $\eta_{0}$ ). This biasing impact can be avoided for the 
Table 1 | The collection of basic "dynamic" characteristics parameters for the tested glass forming materials, Glass forming systems analyzed in the given research report. Footnotes close to short names of compounds are for references recalling experimental data sources. Numbers in parentheses " ()$^{\prime}$ " denote the value of the fitting errors. The parameters $n$ and $T_{N}$ are for the "symmetry-related" exponent $n$ and the singular temperature $\mathrm{T}_{N}$ determined via the linear regression fit at $1 / \mathrm{I}_{\mathrm{DO}}(\mathrm{T})$ plot. The glass transition temperature was estimated using the empirical condition $\tau\left(T_{g}\right)=100 \mathrm{~s}$. The Angell fragility index and the new metric parameters are denoted by $m$ and $M$ respectively. The last column (R) gives the range $\left(T_{g}-T_{\text {end }}\right)$ of tested experimental data

\begin{tabular}{lllrrrrrr} 
Sym. & System & \multicolumn{1}{c}{ Full name } & $T_{g} / K$ & \multicolumn{1}{c}{$T_{N} / K$} & \multicolumn{1}{c}{$n$} & $m$ & $M$ & $R / K$ \\
\hline$O$ & $\mathrm{CNadm}^{34}$ & Cyanoadamantane (ODIC) & 154 & $143(3)$ & $0.14(0.06)$ & 23 & $0.17(0.08)$ & $183-298$ \\
$\square$ & ${\mathrm{C} 8 \mathrm{c} 7^{35}}^{35}$ & Cycloheptanol(57\%) + Cyclooctanol(43\%) (ODIC) & 149 & $119(2)$ & $0.40(0.08)$ & 28 & $0.98(0.09)$ & $155-233$ \\
$\triangleleft$ & $\mathrm{CNC6}^{36}$ & Cyanocyclohexane (ODIC) & 134 & $120(2)$ & $0.14(0.08)$ & 48 & $1.69(0.07)$ & $138-277$ \\
$\Delta$ & Ethnn $^{37}$ & Ethanol (LMW) & 99 & $72(2)$ & $1.23(0.09)$ & 52 & $2.75(0.08)$ & $96-250$ \\
$D$ & $8 * \mathrm{OCB}^{38}$ & Isooctylcyanobiphenyl (LC) & 221 & $190(3)$ & $1.51(0.08)$ & 85 & $4.74(0.06)$ & $224-413$ \\
$\square$ & $\mathrm{PC}^{39}$ & Propylene carbonate (LMW) & 157 & $132(2)$ & $1.13(0.09)$ & 90 & $5.32(0.06)$ & $159-370$ \\
\hline
\end{tabular}

new fragility metric $M=I_{D O}\left(T_{g}\right)=\Delta H_{a}\left(T_{g}\right) / \Delta E_{a}\left(T_{g}\right)-1$, ranging from $M=0$ (the basic Arrhenius case) to $M>10$ for strongly SA dynamics. The activation enthalpy can be easily calculated via $\Delta H_{a}^{\prime}(T)=\Delta H_{a}(T) / R=d \ln \tau(T) / d(1 / T)$ or $\Delta H_{a}^{\prime}=d \ln \eta(T) / d(1 / T)$ and the activation energy via the recently proposed model free route procedure (see Methods and Refs. 29, 30). This report shows that in the ultraviscous/ultraslowing domain always $\Delta H_{a}(T)>\Delta E_{a}(T)$ and in the immediate vicinity of $T_{g}$ even $\Delta H_{a}(T) \gg$ $\Delta E_{a}(T)$. We emphasize this issue, since in a number of research reports the erroneous assumption that $\Delta E_{a}^{\prime}(T)=d \ln \tau(T) / d(1 / T)$ near the glass transition has been used $\mathrm{d}^{20-23,43-49}$.

One of the most attracting questions regarding fragility is its maximal value. In Ref. 50 value $m \approx 175$ was indicated as the maximal possible fragility, what is related to $M \approx 10$. However, earlier $m \approx$ 214 was noted as the indicator of the most SA dynamics ${ }^{51}$. Basing on this report, recalling the basic Adam-Gibbs (AG) theory ${ }^{2,52}$ and Refs. 29, 30 the following general dependence for the apparent activation energy temperature index can be obtained (see Methods section):

$$
I_{D O}(T)=\frac{n T_{N}}{T-T_{N}}
$$

The latter dependence and eq. (9) yields:

$$
M=n \frac{T_{N}}{T_{g}-T_{N}} \quad \text { and } \quad m=C\left(1+n \frac{T_{N}}{T_{g}-T_{N}}\right)
$$

For example, for glass formers with rod-like molecules and the clear uniaxial, orientational symmetry $n \approx 1.6$ and $T_{g}-T_{N} \approx 10$ with $T_{N}$ $\approx 300 \mathrm{~K}$ (see Suppl. Info to Ref. 30) one obtains $m \approx 280$ !

Following the given report and Refs. 29,30 one can postulate that the transformation of $\tau(T)$ or $\eta(T)$ experimental data to $I_{D O}(T)$ representation can yield all basic characteristics of previtreous dynamics, basing solely on inherently unambiguous linear regression fit, namely: (i) the local symmetry related parameter $n=-1 / I_{D O}^{-1}(0)$, (ii) the extrapolated singular temperature for which $I_{D O}^{-1}\left(T_{N}\right)=0$ and (iii) the fragility metric $M=I_{D O}\left(T_{g}\right)$, linked to basic process energies. Knowing $\left(T_{N}, M, n\right)$ (see Fig. 6 in Methods section) and the fragility $m$ (from the "Angell" plot) the unambiguous estimation of $\tau_{0}$ or $\eta_{0}$ prefactors is also possible.

One of still mysterious features of the glass transition are different distances between the glass temperature $\left(T_{g}\right)$ and the extrapolated Kauzmann temperature $\left(T_{K}\right)$ in various glass formers ${ }^{2,53}$. Following the finding the $T_{N}=T_{K}$ (see Ref. 30 and Methods section) and eq.

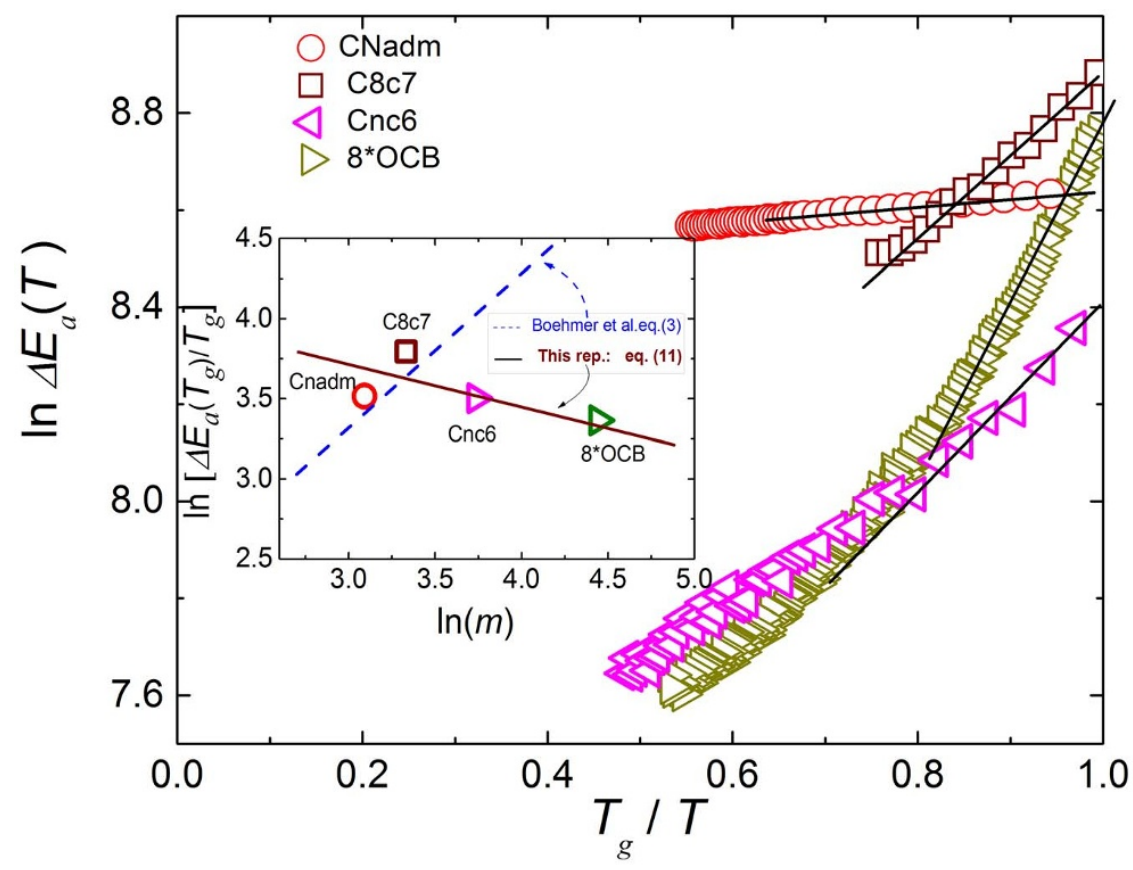

Figure $3 \mid$ Degree of nonlinearity at "Arrhenius-type" plotted as $\ln \left(\Delta E_{a}(T)\right)$ vs. $T_{g} / T$ for representative glass formers. The figure indicates the lack of correlation between increasing curvature, coupled to fragility, and the value of $\Delta E_{a}\left(T_{g}\right)$. The clear disagreement with eq. (3) is stressed by the inset: the blue, dashed line is related to eq. (3) and the solid, black line is based on the MFR. 


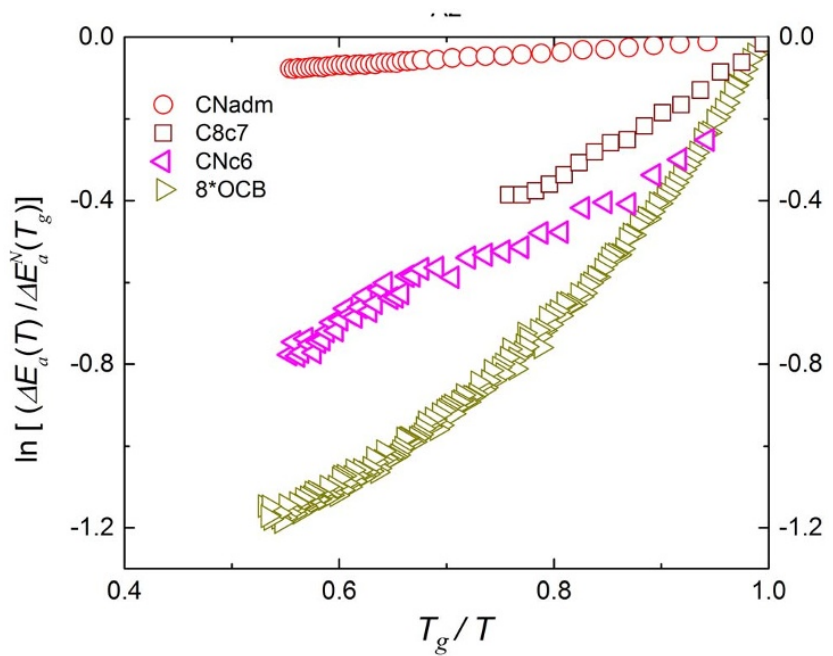

Figure 4 |The "universal" scaling plot for activation energies in representative glass formers. The apparent activation energy is obtained by $\mathrm{MFR}^{29,30}$ procedure (see Methods) which is rescaled at $T_{g}$ using eq. (11). The figure shows the correlation between increasing curvature, indicating the rise of fragility, and $\Delta E_{a}\left(T_{g}\right)$. For basic data see also Table 1.

(13) one obtains $M / n=T_{N} /\left(T_{g}-T_{N}\right)=M / n$, i.e. the relative distance between $T_{K}\left(=T_{N}\right)$ and $T_{g}$ depends solely on the fragility $(M)$ and symmetry related $(n)$. It is notable that eq. (13) makes it possible also unequivocal calculation the $\tau_{0}$ for the SA eq. (2), avoiding any "ersatz equations", like the $\mathrm{VFT}^{2}$ one used so far.

Worth noting is the relationship of the new fragility parameter $M$ to the one introduced by Doremus ${ }^{54}$, who applied the empirical finding that at high temperature (HT) the activation energy low $\left(\Delta E_{a}^{H T}\right.$, originally denoted as $Q_{L}$ is low) whereas at low temperatures prior to the vitrification its value is high $\left(Q_{H}, \Delta E_{a}^{L T}\right)$. This lead to the Doremus fragility metric $R_{D}=Q_{H} / Q_{L}=\Delta E_{a}^{L T} / \Delta E_{a}^{H T}$ with $R_{D}<2$ for "strong" glass formers and $R_{D}>2$ for "fragile" ones ${ }^{2,54}$. In the Doremus model $Q_{H}=H_{d}+H_{m}$, where $H_{d}$ is associated with enthalpy of formation of broken bonds and $H_{m}$ is responsible for motions. At high temperature $H_{m}$ dominates and $Q_{L}=H_{m}$. Such picture results from the fact that Doremus introduced the model for ultraviscous silica, but its extension to other glass formers can be explained by the formations of bond-ordering local structures or heterogeneities. This led Doremus to the double-exponential function for description of the viscous flow, at least in silicates ${ }^{54}$. This report correlates with some fundamental of Doremus model ${ }^{54}$, although the analysis avoids any model assumptions, basing solely on the general Super-Arrhenius equation (eq. (2)).

It is also notable that results of the given report and empirical findings in Refs. 29, 30 lead to surprisingly simple form of relative changes of process energies in the ultraviscous/ultraslowing domain $\Delta E_{a}(T) /\left[\Delta H_{a}(T)-\Delta E_{a}(T)\right]=a+b T$ with $a \neq 0$ and $b \neq 0$.

Concluding, this reports presents the link between fragility and basic process energies, in ultraviscous/ultraslowing glass forming materials lacking so far. However, results presented above are also significant for complex systems and collective phenomena if their dynamics is described by the general super-Arrhenius relation.

\section{Methods}

The analysis of experimental data and the model-free route (MFR) method. The "model-free" route procedure introduced in Refs. 29, 30 is a novel approach for getting insight into previtreous dynamics based on the transformation of basic structural relaxation time $(\tau(T))$ or viscosity $(\eta(T))$ experimental data to apparent activation energy temperature index form, the magnitude first proposed for glass formers by Dyre and Olsen $(\mathrm{DO})^{40}$ via:

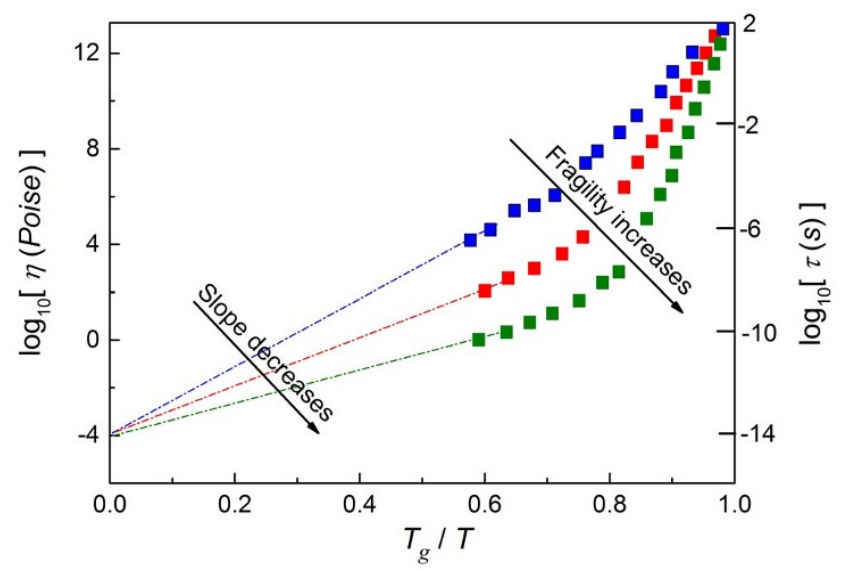

Figure 5 The schematic plot explaining the source of the hypothetical correlation between fragility $m$ and the activation energy for the high temperature border case $T_{g} / T \rightarrow 0$. Fragility is related to the slope for $T_{g} / T$ $\rightarrow 1$, in the ultraviscous, "low temperature", dynamic domains (indicated by colored symbols). Lines are for the high temperature domain terminating at $T_{g} / T \rightarrow 0$. The plot recalls the basis of Novikov and Sokolov ${ }^{27,28}$ relationship linking fragility and activation energy in the high temperature domain (eq. (4)). The presumable "universal" high temperature $\left(T_{g} / T \rightarrow 0\right)$ values of prefectors in SA eq. (2) are $\eta_{0}=10^{-4}$ Poise or $\tau_{0}=10^{-14} s^{2}$.

$$
I_{D O}(T)=-\frac{d \ln \Delta E_{a}(T)}{d \ln T}
$$

The apparent activation energy $\Delta E_{a}^{\prime}(T)=\Delta E_{a}(T) / R$ is determined from $\tau(T)$ and $\eta(T)$ experimental data via the solution of the differential equation resulting from the general SA eq. $(2)^{29,30}$ :

$$
\frac{\partial \Delta E_{a}^{\prime}(T)}{\partial(1 / T)}+\frac{\Delta E_{a}^{\prime}(T)}{(1 / T)}=\frac{\Delta H_{a}^{\prime}(T)}{(1 / T)}
$$

where the apparent activation enthalpy is given by $\Delta H_{a}^{\prime}(T)=\Delta H_{a} / R=d \ln \tau(T) /$ $d(1 / T)^{29}$.

This way of determining $\Delta E_{a}^{\prime}(T)$ was only recently introduced in Refs. 29, 30. Previously, the apparent activation energy was calculated from the SA eq.(2) as $\Delta E_{a}(T)=R T \ln \left(\tau(T) / \tau_{0}\right)^{2,40,41}$. and then it was inherently biased by the generally unknown estimation of $\tau_{0}$ prefactor ${ }^{2}$. In practice, a "universal" value of $\tau_{0}=10^{-14} \mathrm{~s}$ was most commonly assumed ${ }^{2,40,41}$. An inherent advantage of the MFR approach for determining $\Delta E_{a}(T)$ and $I_{D O}(T)$ includes also the application of a numerical filtering procedure based on Savitzky-Golay principle ${ }^{29,30}$.

In Refs. 29, 30 the MFR have been implemented for a set of 55 glass forming systems, ranging from low molecular weight liquids (LMW) and polymers (P) to liquid crystal (LC), plastic crystal (ODIC) and spin glasses (SGL), in the previtreous domain. The analysis revealed a surprisingly simple pattern for the previtreous dynamics: $1 / I_{D O}(T)=a T+b$, with $a \neq 0$ and $b \neq 0$ for all mentioned data sets. This led to the derivation of the new generalized configurational entropy equation $S_{c}(T)=$ $S_{0}\left(1-\left(T_{N} / T\right)\right)^{n}$, where the power exponent is determined as

$n=-(1 / b)=-1 / I_{D O}^{-1}(T=0)$ and $T_{N}$ is the singular temperature estimated via $I_{D O}\left(T=T_{N}\right)^{-1}=0^{29,30}$. Recently, basing on the MFR, the clear coincidence between the ideal glass (Kauzmann) temperature $T_{K}$ and $T_{N}$, i.e. $T_{K}=T_{N}$, was found ${ }^{30}$. The analysis carried out in Refs. 29,30 revealed that the parameter ranges between $0.18<$ $n<1.53$, where the lower limit is for systems with the clear positional symmetry (PS) and the higher one for systems with clear orientational, uniaxial symmetry (OS). The dynamics of PS and OS glass formers is relatively well portrayed by the critical-like equation ${ }^{29,38}$. The third characteristic case is for systems where $n=1$ (no-symmetry). Only in this case the application of the popular Vogel-Fulcher-Tammann (VFT) ${ }^{2}$ equation is suitable ${ }^{29,30}$. Consequently, the fundamental justification of the VFT relation is limited to a small group of glass formers and otherwise (i.e. for $n \neq 1$ ) it can be considered solely as an effective fitting tool.

The example of analysis employing the MFR analysis, based on transformed $\tau(T)$ experimental data in supercooled liquid crystalline $n$-octyloxycyanobiphenyl $\left(8^{*} \mathrm{OCB}\right)$, is shown in Fig. 6 . The way of determining the basic parameters is indicated.

Values of primary relaxation times $\tau(T)$ were determined as the reciprocal of the peak frequency of $\varepsilon^{\prime \prime}(f)$ loss curve, obtained from broad band dielectric spectroscopy measurement (see Refs. 29, 30). 


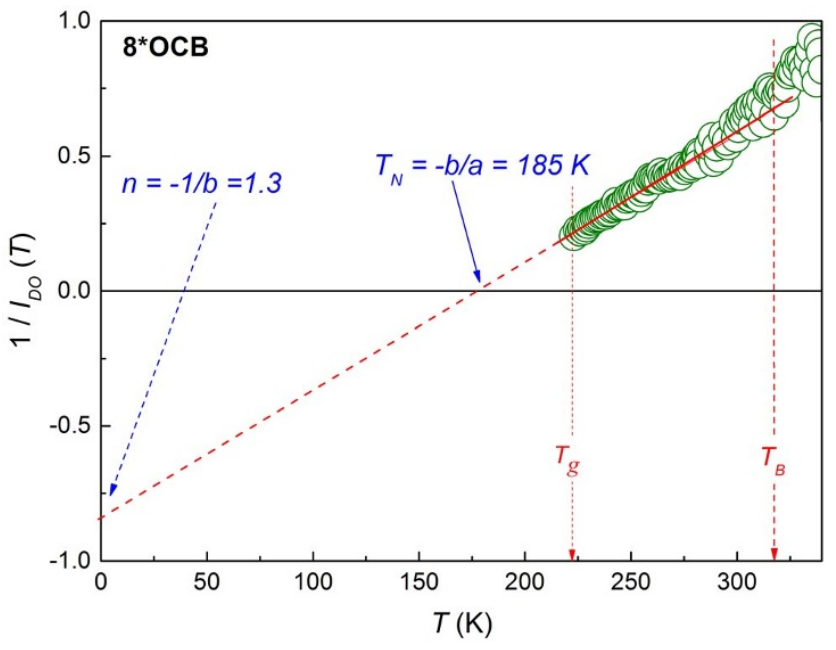

Figure 6 | The temperature scaling behavior of the reciprocal of the apparent activation temperature index in glass forming liquid crystalline n-octylocycyanobiphenyl (8*OCB). The ultraviscous domain extends between $T_{g}<T(\approx 100 \mathrm{~K})<T_{B}$, where the latter denotes the dynamic crossover temperature ${ }^{2}$. Locations of the singular temperature $T_{N}$ and the extrapolation down to $T=0$ as well as the method of calculating the experimental dependence $I_{D O}^{-1}=a+b T$ are shown ${ }^{29,30}$.

Derivation of the general form for the activation energy temperature index (eq (12). Recalling the Adam-Gibbs theory for glass transtion ${ }^{2,52}$, the apparent activation index can be written as ${ }^{29,30}$ :

$$
I_{D O}(T)=-\frac{d \ln \Delta E_{a}(T)}{d \ln T}=\frac{T d \ln S_{c}(T)}{d T}=\frac{T}{S_{c}(T)} \frac{d S_{c}(T)}{d T}
$$

Substituting the new generalized configurational entropy (Ref. 29) $S_{c}(T)=S_{0}(1-$ $\left.\left(T_{N} / T\right)\right)^{n}$, one obtains:

$$
\begin{aligned}
I_{D O}(T) & =\frac{T}{S_{c}(T)}\left[\frac{n T_{N} S_{o}\left(1-\left(T_{N} / T\right)\right)^{n}}{\left(1-\left(T_{N} / T\right)\right) T^{2}}\right]=\frac{S_{o}\left(1-\left(T_{N} / T\right)\right)^{n}}{S_{c}(T)}\left[\frac{n T_{N}}{\left(T-T_{N}\right)}\right]= \\
& =\frac{n T_{N}}{\left(T-T_{N}\right)}
\end{aligned}
$$

The above dependence make it possible to identify the impact of the entropic contribution in the anomalous behavior of the activation entropy temperature index. Alternatively eq. (12) can be derived recalling the experimental finding that $1 /$ $I_{D O}(T)=a T+b$, where $b=(-1 / n) \neq 0$ and $a=\left(1 / n T_{N}\right) \neq 0$ (the coefficient $n=-(1 / b)=-1 / I_{D O}^{-1}(T=0)$ and the singular temperature $\left.I_{D O}\left(T=T_{N}\right)^{-1}=0\right)^{29,30}$, one obtains $\Delta H_{a}(T) / \Delta E_{a}(T)=\left[T_{N}(n-1)+T\right] /\left(T-T_{N}\right)$ and then $I_{D O}(T)=n T_{N} /(T$ $\left.-T_{N}\right)$.

1. Kennedy, D. 125 outstanding problems in all of science: What is the nature of the glassy state. Science 309, 83 (2005).

2. Ngai, K. L. Relaxation and Diffusion in Complex Systems (Springer, Berlin, 2011).

3. Stillinger, F. H. \& Debenedetti, P. G. Glass transitions: thermodynamics and kinetics. Annu. Rev. Cond. Matt. Phys. 4, 262-270 (2013).

4. Jones, R. A. L. Soft condensed matter (Oxford Univ. Press., Oxford, 2002).

5. Mauro, J. C. Glass: The nanotechnology connection. Int. J. Appl. Glass Sci. 4, 64-75 (2013).

6. Thomas, S. A. \& Zachariah, K. Polymer Processing and Characterization (Apple Academic Press, Boca Raton (FL, USA) 2012).

7. Jadhav, N. R., Gaikwad, V. L., Nair, K. J. \& Kadam, H. M. Glass transition temperature: Basics and application in pharmaceutical sector. Asian J. Pharm. 3 , 82-89 (2009).

8. Roos, Y. H. Glass transition temperature and its relevance in food processing. Ann. Rev. Food Sci.\& Technol. 1, 469-496 (2010).

9. Starzonek, S., Rutkowska, M., Rzoska, S. J., Drozd-Rzoska, A. \& Fonberg-Broczek et al. Dielectric spectroscopy of pressurized Saccharomyces cerevisiae, Food Biophysics 362, 1-8 (2014).

10. Poirier, J. P. Introduction to physics of the Earth interior (Cambridge Univ. Press, Cambridge, 2000).

11. Schroers, J. Condensed-matter physics: Glasses made from pure metals, Nature 512, 142 (2014)

12. Singh, J. \& Shimagawa, K. Advances in Amorphous semiconductors (CRC Press, London, 2003)
13. Mallamace, F. \& Stanley, H. E. The physics of complex systems (New advances and perspectives) (IOS Press, Amsterdam, 2004).

14. Angell, C. A. Strong and fragile liquids, in: Relaxations in Complex Systems, eds. Ngai, K. L. \& Wright, G. B. (National Technical Information Service, U.S. Dept. of Commerce, Springfield,1985).

15. Boehmer, R., Ngai, K. L., Angell, C. A. \& Plazek, J. D. Nonexponential relaxations in strong and fragile glass formers. J. Chem. Phys. 99, 4201-4209 (1993).

16. Zheng, Q., Mauro, J. C., Ellison, A. J., Potuzak, M. \& Yue, Y. Universality of the high-temperature viscosity limit of silicate liquids, Phys. Rev. B 83, 212202 (2011).

17. Drozd-Rzoska, A. \& Rzoska, S. J. On the derivative-based analysis for temperature and pressure evolution of dielectric relaxation times in vitrifying liquids. Phys. Rev. E 73, 041502 (2006).

18. Greer, A. L., Kelton, K. F. \& Sastry, S. (eds.). Fragility of glassforming liquids (Hindustan Book Agency, Bengaluru, 2014).

19. Kivelson, S. A. \& Tarjus, G. In search of a theory of supercooled liquids. Nature Mater. 7, 831-833 (2008).

20. Qin, Q., McKenna, G. B. Correlation between dynamic fragility and glass transition temperature for different classes of glass forming liquids, J. Non-Cryst. Solids 352, 2977-2985 (2006).

21. Moura Ramos, J. J., Correia, N. T., Taveira-Marques, R. \& Collins, G. The activation energy at $T_{g}$ and the fragility index of indomethacin, predicted from the influence of the heating rate on the temperature position and on the intensity of thermally stimulated depolarization current peak, Pharm. Res. 19, 1879-1884 (2002).

22. Zhao, J. \& McKenna, G. B. The apparent activation energy and dynamic fragility of ancient ambers, Polymer 55, 2246-2253 (2014).

23. Soltan, A. S., Abu-Sehly, A. A., Joraid, A. A. \& Alamri, S. N. The activation energy and fragility index of the glass transition in $\mathrm{Se}_{76} \mathrm{Te}_{21} \mathrm{Sb}_{3}$ chalcogenide glass, Thermochimica Acta 574, 73-78 (2013).

24. Vyazovkin, S., Sbirrazzuoli, N. \& Dranca, I. Variation in activation energy of the glass transition for polymers of different dynamic fragility, Macromol. Chem. Phys. 207, 1126-1130 (2006).

25. Saffarini, G., Saiter, Ã. A., Garda, M. R. \& Saite, J. M. Mean-coordination number dependence of the fragility in Ge-Se-In glass-forming liquids, Physica B 389 275-280 (2007).

26. Aniya, M. Understanding the composition dependence of the fragility of AgI Ag2O-MxOy glassy systems, IOP Conf. Series: Materials Science and Engineering 18, 112003 (2011).

27. Novikov, V. N. \& Sokolov, A. P. Poisson's ratio and the fragility of glass-forming liquids, Nature 431, 961-963 (2004).

28. Novikov, V. N., Ding, Y., \& Sokolov, A. P. Correlation of fragility of supercooled liquids with elastic properties of glasses, Phys. Rev. E 71, 061501 (2005).

29. Martinez-Garcia, J. C., Rzoska, S. J., Drozd-Rzoska, A. \& Martinez-Garcia, J. A. universal description of ultraslow glass dynamics. Nat. Commun. 4, 1823-1830 (2013).

30. Martinez-Garcia, J. C., Rzoska, S. J., Drzozd-Rzoska, A., Martinez-Garcia, J. \& Mauro, J. C. Divergent dynamics and the Kauzmann temperature in glass forming systems, Sci. Rep. 4, 5160 (2014).

31. Vogel, H. Temperaturabhangigkeitsgesetz der viskositaet von fluessigkeiten. Phys. Zeit. 22, 645-646 (1921).

32. Fulcher, G. S. Analysis of recent measurements of the viscosity of glasses. J. Am. Ceram. Soc. 8, 339-355 (1925).

33. Tammann, G. Glasses as supercooled liquids. J. Soc. Glass Technol. 9, 166-185 (1925).

34. Martinez-Garcia, J. C., Tamarit, J. Ll., Capaccioli, S., Barrio, M. \& Veglio, N. et al. $\alpha$-relaxation dynamics of orientanionally disordered mixed crystals composed of $\mathrm{Cl}$-adamantane and $\mathrm{CN}$-adamantane. J. Chem. Phys. 132, 164516-164526 (2010).

35. Martinez-Garcia, J. C., Tamarit, J. Ll., Pardo, L. C., Barrio, M. \& Rzoska, S. J. et al. A. Disentangling the secondary relaxations in the orientationally disordered mixed crystals: cycloheptanol + cyclooctanol two-component system, J. Phys. Chem. B 114, 6099-6106 (2010).

36. Martinez-Garcia, J. C., Tamarit, J. Ll., Rzoska, S. J., Drozd-Rzoska, A. \& Pardo, L. C. et al. Universal critical-like scaling of dynamics plastic crystals. J. Non-Cryst. Solids 357, 329-333 (2011).

37. Brand, R., Lunkenheimer, P., Schneider, U. \& Loidl, A. Excess wing in the dielectric loss of glass-forming ethanol: A relaxation process. Phys. Rev. B 62 , 8878-8883 (2000).

38. Drozd-Rzoska, A., Rzoska, S. J. \& Paluch, M. Universal critical-like scaling of dynamic properties in symmetry-selected glass formers. J. Chem. Phys. 129, 184509-184515 (2008).

39. Stickel, F. "Untersuchungen der dynamik in niedermolekularen flüssigkeiten mit dielektrischer spektroskopie", PhD. dissertation (Mainz University, 1995).

40. Dyre, J. C. \& Olsen, N. B. Landscape equivalent of the shoving model. Phys. Rev. E 69, 042501 (2004).

41. Hecksher, T., Nielsen, A. I., Olsen, N. B. \& Dyre, J. C. Little evidence for dynamic divergences in ultraviscous molecular liquids. Nature Phys. 4, 737-741 (2008).

42. Agapov, A. L., Novikov, V. N. \& Sokolov, A. P. Fragility and other properties of glass-forming liquids: Two decades of puzzling correlations, in Greer, L. A., Kelton, K., Sastry, S. (eds.), Fragility of glass forming liquids (Hindustan Book Agency, 2013). 
43. Debenedetti, P. G. \& Stillinger, F. H. Supercooled liquids and the glass transition, Nature 410, 259-267 (2001).

44. Capaccioli, S., Lucchesi, M., Rolla, P. A. \& Ruggeri, G. Dielectric response analysis of a conducting polymer dominated by the hopping charge transport, J. Phys.: Condens. Matter 10, 5595-5617 (1998).

45. Macovez, R., Zachariah, M., Romanini, M., Zygouri, P. \& Gournis, D. et al. Hopping conductivity and polarization effects in a fullerene derivative salt, J. Phys. Chem. C 118, 12170-12175 (2014).

46. Monkos, K. Analysis of the viscosity-temperature-concentration dependence for dimeric bovine $\beta$-lactoglobulin aqueous solutions on the basis of the Vogel-Tammann-Fulcher's equation, Current Topics in Biophys. 31, 16-24 (2008).

47. Viciosa, M. T. \& Dionısio, M. Molecular mobility and fragility in n-ethylene glycol dimethacrylate monomers, J. Non-Cryst. Solids 341, 60-67 (2004).

48. Jadżyn, J., Czechowski, G., Legrand, C. \& Douali, R. Static and dynamic dielectric properties of strongly polar liquids in the vicinity of first order and weakly first order phase transitions. Phys. Rev. E 67, 041705 (2003).

49. Paluch, M., Roland, C. M., Casalini, R., Meier, G. \& Patkowski, A. The relative contributions of temperature and volume to structural relaxation of van der Waals molecular liquids. J. Chem. Phys. 118, 4578-4582 (2003)

50. Wang, L.-M. \& Mauro, J. C. An upper limit to kinetic fragility in glass-forming liquids. J. Chem. Phys. 134, 044522 (2011).

51. Simon, S. L., Plazek, D. J., Sobieski, J. W. \& McGregor, E. T. Physical aging of a polyetherimide: Volume recovery and its comparison to creep and enthalpy measurements. J. Polym. Sci. Part B. Polym. Phys. 35, 929-936 (1997).

52. Adam, G. \& Gibbs, J. H. On the temperature dependence of cooperative relaxation properties in glass-forming liquids. J. Chem. Phys. 43, 139-146 (1965).

53. Tanaka, H. Relation between thermodynamics and kinetics of glass-forming liquids. Phys. Rev. Lett. 90, 05570 (2003).
54. Doremus, R. H. J. Viscosity of silica, J. Appl. Phys. 92, 7619-7630 (2002).

\section{Acknowledgments}

This research was carried out due to the support of the National Centre for Science (Poland), for SJR via grant UMO-2011/03/B/ST3/02352 and for ADR via grant UMO-2011/ 01/B/NZ9/02537.

\section{Author contributions}

J.C.M.G., S.J.R. and A.D.R. wrote the main manuscript, J.C.M.G. prepared figures, S.z.S. worked on data analysis, J.C.M. finally shaped the form of the manuscript. Authors' related experimental results are associated with measurements carried out J.C.M.G., S.J.R. and A.D.R. New conceptions proposed in the paper are proposed mainly by J.C.M.G. and S.J.R but finally influenced by A.D.R. and J.C.M.

\section{Additional information}

Competing financial interests: The authors declare no competing financial interests.

How to cite this article: Martinez-Garcia, J.C., Rzoska, S.J., Drozd-Rzoska, A., Starzonek, S. \& Mauro, J.C. Fragility and basic process energies in vitrifying systems. Sci. Rep. 5, 8314; DOI:10.1038/srep08314 (2015)

This work is licensed under a Creative Commons Attribution 4.0 International License. The images or other third party material in this article are included in the article's Creative Commons license, unless indicated otherwise in the credit line; if the material is not included under the Creative Commons license, users will need to obtain permission from the license holder in order to reproduce the material. To view a copy of this license, visit http://creativecommons.org/licenses/by/4.0/ 